\title{
Human immunodeficiency virus infection induces lymphoid fibrosis in the BM-liver- thymus-spleen humanized mouse model
}

\author{
Jasmine Samal, Samantha Kelly, Ali Na-Shatal, Abdallah Elhakiem, Antu Das, Ming Ding, \\ Anwesha Sanyal, Phalguni Gupta, Kevin Melody, Brad Roland, Watfa Ahmed, Aala Zakir, \\ and Moses Bility \\ Department of Infectious Diseases and Microbiology, University of Pittsburgh, Pittsburgh, Pennsylvania, USA.
}

\begin{abstract}
A major pathogenic feature associated with HIV infection is lymphoid fibrosis, which persists during antiretroviral therapy (ART). Lymphoid tissues play critical roles in the generation of antigenspecific immune response, and fibrosis disrupts the stromal network of lymphoid tissues, resulting in impaired immune cell trafficking and function, as well as immunodeficiency. Developing an animal model for investigating the impact of HIV infection-induced lymphoid tissue fibrosis on immunodeficiency and immune cell impairment is critical for therapeutics development and clinical translation. Said model will enable in vivo mechanistic studies, thus complementing the wellestablished surrogate model of SIV infection-induced lymphoid tissue fibrosis in macaques. We developed a potentially novel human immune system-humanized mouse model by coengrafting autologous fetal thymus, spleen, and liver organoids under the kidney capsule, along with i.v. injection of autologous fetal liver-derived hematopoietic stem cells, thus termed the BM-liverthymus-spleen (BLTS) humanized mouse model. BLTS humanized mouse model supports development of human immune cells and human lymphoid organoids (human thymus and spleen organoids). HIV infection in BLTS humanized mice results in progressive fibrosis in human lymphoid tissues, which was associated with immunodeficiency in the lymphoid tissues, and lymphoid tissue fibrosis persists during ART, thus recapitulating clinical outcomes.
\end{abstract}

License: This work is licensed under the Creative Commons Attribution 4.0 International License. To view a copy of this license, visit http:// creativecommons.org/licenses/ by/4.0/.

Conflict of interest: The authors have declared that no conflict of interest exists.

Submitted: February 19, 2018 Accepted: August 7, 2018 Published: September 20, 2018

Reference information: JCI Insight. 2018;3(18): e120430. https://doi.org/10.1172/jci. insight.120430.

\section{Introduction}

The hallmark of chronic HIV infection is the depletion of $\mathrm{CD}^{+} \mathrm{T}$ cells, via direct virus-mediated killing and indirect killing, which ultimately leads to AIDS in the absence of antiretroviral therapy (ART). Inflammatory response is also a hallmark of chronic HIV infection, and studies suggest that HIV-induced inflammatory response results in lymphoid tissue fibrosis, which contributes to lymphoid tissue damage, $\mathrm{CD}^{+} \mathrm{T}$ cell depletion, and impaired $\mathrm{CD}^{+}{ }^{+} \mathrm{T}$ cell reconstitution following ART (1-7). A study also demonstrated that HIV-associated lymphoid tissue fibrosis is present in all categories of HIV-infected patients, including HIV controllers and noncontrollers with or without ART (8). A notable exception is the so-called Berlin patient, the only recorded case of complete and sustained remission of HIV; collagen levels in lymphoid tissues of the Berlin patient were similar to healthy, uninfected controls (8).

Lymphoid tissues play a critical role in the generation of antigen-specific immune cells; thus, damage to these organs significantly impairs the generation of robust host-immune response to infectious agents and microbial antigens. Immune cells/therapeutic vaccine-based therapies for sustained HIV remission or eradication is predicated on the notion that infused or vaccine-induced immune cells will control or eradicate the HIV reservoir, which includes the HIV reservoir located in lymphoid tissues (9). This idea posits that infused or vaccine-induced HIV-targeting immune cells will survive and maintain functionality in the fibrotic lymphoid tissues; however, there are no current small animal models for HIV-induced lymphoid tissue fibrosis to evaluate said notion.

Studies using the surrogate SIV macaque model demonstrate that pathogenic SIV infection induces progressive lymphoid tissue fibrosis, which strongly correlates with $\mathrm{CD} 4^{+} \mathrm{T}$ cell depletion $(2,7,10)$; however, virus/species-specific limitations may exist in translating findings from mechanistic studies to HIV-human host interactions (11). Humanized mice reconstituted with human immune cells with or without human 
thymus organoid (primary lymphoid organ), broadly termed human immune system-humanized mice, has been widely used to study HIV transmission, immunopathogenesis, chronic inflammation, and immune response and is highly amendable to mechanistic studies, thus providing a robust small animal model that complements the surrogate SIV macaque model (12). In human immune system-humanized mouse models, the immunodeficient mouse spleen is reconstituted with human immune cells to generate a humanized murine spleen; however, human immune cell reconstitution and associated red pulp and lymphoid follicle development in the humanized murine spleen is not optimal (13). The suboptimal human immune cell reconstitution of the humanized murine spleen in human immune system-humanized mouse models is exhibited by the disorganized splenic architecture and associated low red pulp macrophage reconstitution, which - in contrast - is the most abundant cell type in the human spleen (14).

To improve the human lymphoid organoid system in human immune system-humanized mice, we coengrafted autologous fetal thymus, spleen, and liver organoids under the kidney capsule, along with i.v. injection of autologous fetal liver-derived hematopoietic stem cells, thus generating BM-liver-thymus-spleen (BLTS) humanized mice. The premise for this approach is well established, as several reports have demonstrated development of spleen xenograft under the kidney capsule in immunodeficient mice (15-17). The BLTS humanized mouse model supports human immune cell development, along with development of human thymus and spleen organoids, which are comparable with human lymphoid organs in terms of both lymphoid tissue architecture and cellular composition and distribution. We investigated HIV-induced collagen deposition (fibrosis) in human thymus and spleen tissues in the BLTS humanized mouse model. We demonstrate that HIV infection induces excessive human collagen deposition (fibrosis) in human spleen and thymus tissues in the BLTS humanized mouse model. Understanding the mechanisms of HIV-induced lymphoid tissue fibrosis and its role in chronic inflammation, immune cell impairment, immunodeficiency, and the generation, trafficking, and function of anti-HIV immune cells is critical for developing novel therapeutic strategies for treating persistent immune abnormalities associated with non-AIDS pathologies and eradicating the HIV reservoir; the BLTS humanized mouse model provides a platform for addressing these questions.

\section{Results}

The BLTS humanized mouse model supports development of human spleen and thymus organoids and human immune cells. Reports suggest that the microanatomy of lymphoid tissues plays a critical role in immune cell development, and species-specific differences exist in the microanatomy of human and mouse lymphoid tissues (18-20). The mouse thymus microanatomy differs from human thymus microanatomy by the absence of sublobulation formed by a thin layer of collagen and reticular fibers, which are produced by reticular fibroblasts (19) (Supplemental Figure 1; supplemental material available online with this article; https://doi. org/10.1172/jci.insight.120430DS1). The spleen, which is a major secondary lymphoid tissue accounting for $\sim 25 \%$ of total body lymphocytes, exhibits significant differences in the relative size and structure of the red and white pulp between humans and mice (18) (Supplemental Figure 1). The human spleen has a small white pulp/red pulp area ratio; thus, red pulp macrophages and stromal constituents including reticular fibroblasts and reticular fibers are dominant components of the human spleen $(18,20)$ (Supplemental Figure 1). In contrast, the ratio of white pulp/red pulp area is high in the mouse spleen; thus, the mouse spleen has low level of stromal constituents $(18,20)$ (Supplemental Figure 1). We hypothesized that differences in the microanatomy of human and mouse spleens could contribute to suboptimal development of human immune cells in immunodeficient mouse spleen. We hypothesized that cotransplantation of autologous fetal thymus, spleen, and liver organoids under the kidney capsule - along with i.v. injection of autologous fetal liver-derived hematopoietic stem cells, termed BLTS humanized mice - will enable development of human spleen and thymus organoids and associated human immune cells in the organoids and the periphery (Figure 1). We processed autologous human fetal spleen, thymus and liver organs into $\sim 1 \mathrm{~mm}^{2}$ organoids and isolated autologous human $\mathrm{CD} 34^{+}$hematopoietic stem cells from the fetal liver, and we subsequently transplanted the organoids and stem cells into irradiated NSG mice (Figure 1 and Supplemental Figure 2, A and B). Gross analysis of human lymphoid organoid development in BLTS humanized mice at 10 weeks after transplantation showed development of human spleen and thymus organoids under the kidney capsule (Figure 2A). In addition to supporting the development of human lymphoid organoids, the BLTS humanized mouse model supports reconstitution of the immunodeficient mouse lymph nodes (axillary and mesenteric) and the immunodeficient mouse spleen when compared with the day of transplantation (Figure 2A and Supplemental Figure 3, A-D). Longitudinal analysis also showed gross development 


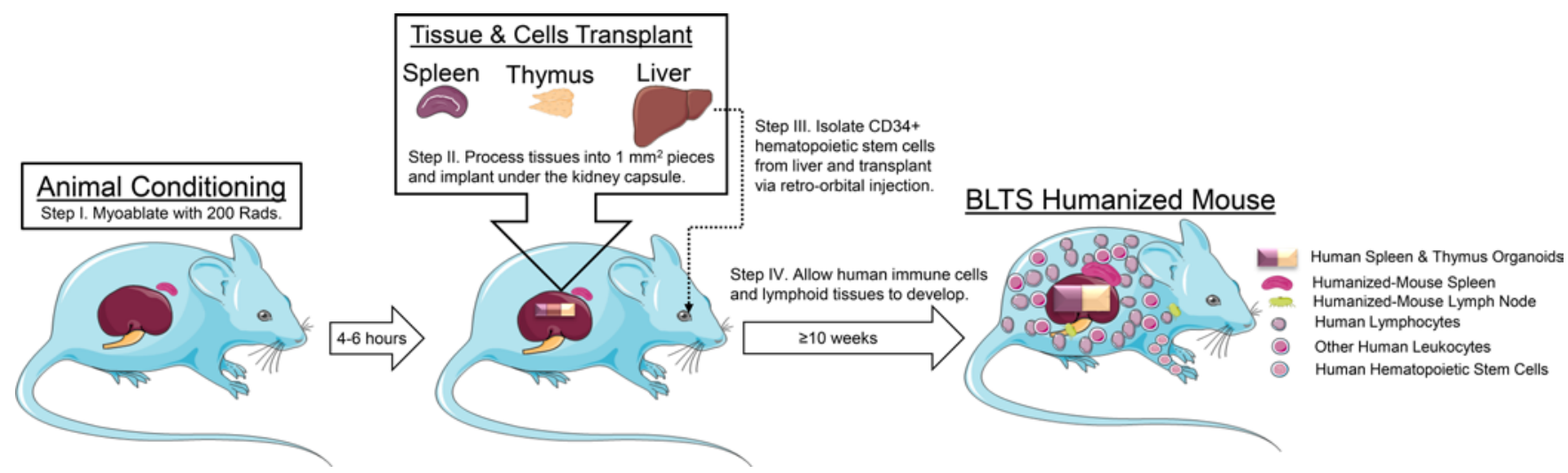

Figure 1. Construction of the BLTS humanized mouse model. In Step I, NSG mice are myoablated via $\gamma$ irradiation (200 rads) using a Cesium-137 irradiator. In Step II, fetal tissues (spleen, thymus, and liver) are processed into $1 \mathrm{~mm}^{2}$ pieces and transplanted as a "sandwich" under the kidney capsule in irradiated NSG mice, following the administration of antibiotic and analogesic therapy and the induction of general anesthesia. In Step III, autologous CD34+ hematopoietic stem cells are isolated from the fetal liver via magnetic selection and transplanted at $2 \times 10^{5}$ cells per mouse via retro-orbital injection following kidney capsule transplantation of the lymphoid tissues. In Step IV, transplanted NSG mice were maintained under specific pathogen free conditions, and the human spleen and thymus organoids - along with other lymphoid tissues and associated immune cells - were allowed to develop over a period of 10 weeks, resulting in the BLTS humanized mouse model.

of human lymphoid organoids (spleen and thymus) (Figure 2B) and gross reconstitution of the immunodeficient mouse spleen and lymph nodes (axillary) (Figure 2, B and C), which are maintained throughout the natural life span (i.e., $\sim 12$ months and 36 weeks after transplantation). Interestingly, cotransplantation of autologous human fetal spleen, thymus, and liver tissues under the kidney capsule, along with transplantation of autologous fetal liver-derived hematopoietic stem cells, resulted in greater than $90 \%$ of BLTS humanized mice without visible symptoms (excessive hair loss and wasting syndrome) of graft versus host diseases (GVHD) as long as 36 weeks after transplantation (Figure 2D). Instances ( $<10 \%)$ of GVHD in the BLTS humanized mouse model were associated with the failure of the human spleen and thymus organoids to coengraft/develop together (data not shown). The absence of GVHD in the BLTS humanized mice contrasts from human immune system-humanized mice reconstituted via cotransplantation of autologous human fetal thymus and liver tissues under the kidney capsule, along with transplantation of autologous fetal liver-derived hematopoietic stem cells, and termed BM-liver-thymus (BLT) humanized mice, which exhibit $>90 \%$ incidence of GVHD by 24 weeks after transplantation (21) (Supplemental Figure 4).

Histochemical analysis of human lymphoid organoids (spleen and thymus) in the BLTS humanized mice demonstrates development of human lymphoid organoids (spleen and thymus), with the microanatomy of the human lymphoid organoids at 10 weeks after transplantation comparable with human lymphoid organs and different from mouse lymphoid organs (Figure 3A and Supplemental Figure 1). Although very little is known about the early development of human thymus and spleen, the features associated with the early development of the thymus and spleen organoids in the BLTS humanized mice at 4 weeks after transplantation are generally consistent with the model of thymus $(22,23)$ and spleen (24) development in mice. In the human thymus, the densely packed heterochromatin of the lymphocyte nuclei forms the cortex and is responsible for the dark blue staining in sections stained with $\mathrm{H} \& \mathrm{E}$; within the cortex resides the medulla, which contains fewer lymphocytes, hence the relatively lighter stain. The human thymus organoid at 10 weeks after transplantation in the BLTS humanized mouse model recapitulates this feature (Figure 3A and Supplemental Figure 1). In the human spleen, the densely packed heterochromatin of the lymphocyte nuclei, which forms the white pulp is responsible for the dark blue staining in sections stained with H\&E; the human spleen organoid at 10 weeks after transplantation in the BLTS humanized mouse model recapitulates this feature (Figure 3A and Supplemental Figure 1). Conversely, the abundance of red blood cells (erythrocytes) and the low levels of lymphocytes in regions surrounding the white pulp accounts for the red stain (red pulp) in human spleen sections stained with H\&E; the human spleen organoid at 10 weeks after transplantation in the BLTS humanized mouse model recapitulates this feature (Figure 3A and Supplemental Figure 1).

The distinct microanatomy of lymphoid tissues results in a distinct immune cell distribution profile in different human lymphoid tissues and plays a critical role in the immune function of the respective 

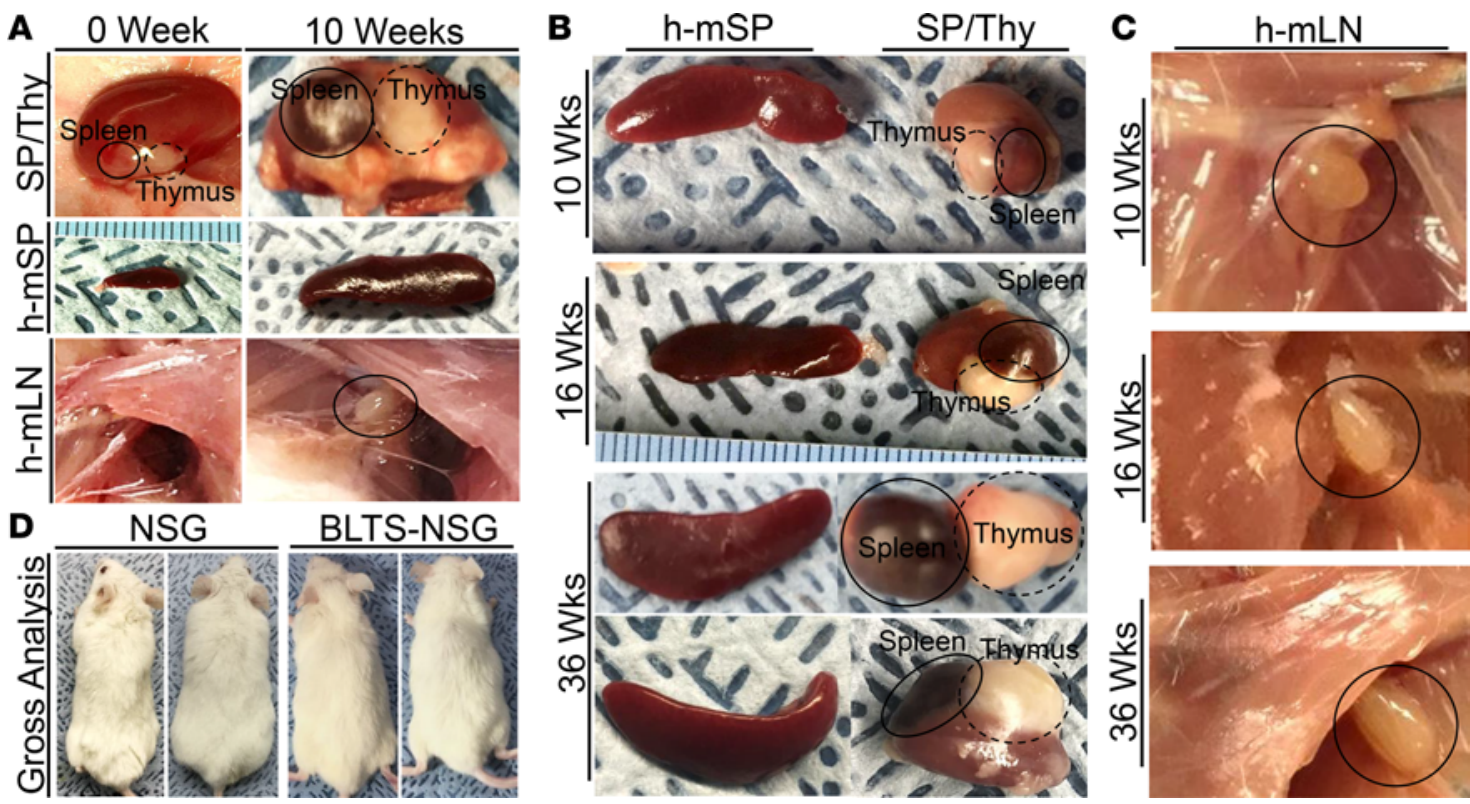

Figure 2. Lymphoid tissues development in the BLTS humanized mouse model. (A) Representative gross analysis of the human spleen and thymus organoids (SP/Thy; images not presented at similar scale) in the kidney capsule, humanized murine spleen (h-mSP; images presented at similar scale), humanized murine lymph nodes (axillary) (h-mLN; images presented at similar scale) in BLTS humanized mice at 0 weeks (day of transplantation) and 10 weeks after transplantation. Representative gross analysis of the (B) humanized murine spleen (h-mSP) and the human spleen and thymus organoids (SP/Thy) in the kidney capsule (images presented at similar scale) and (C) humanized murine lymph nodes (axillary) (h-mLN) in BLTS humanized mice at indicated time points after transplantation. (D) Representative gross analysis of the absence of graft versus host disease (lack of fur loss or wasting syndrome) in age-matched, nontransplanted NSG mice and BLTS humanized NSC mice at 36 weeks after transplantation. Shown are representative gross tissues or animals ( $n=4$ per group). Black circles identifies tissues of interest.

lymphoid organs. We examined the reconstitution and distribution profile of the immune cells in the human lymphoid organoids in the BLTS humanized mouse model. Immunohistochemical analysis of human spleen and thymus organoids in the BLTS humanized mouse model showed human immune cell reconstitution, with a distinct immune cell distribution profile comparable with human lymphoid organs $(18,19)$. Human thymus organoid in the BLTS humanized mice exhibit robust $\mathrm{T}$ cell (human $\mathrm{CD}^{+}$cells) reconstitution, with $\mathrm{T}$ cell levels highest in the cortex and relatively lower in the medulla (Figure $3 \mathrm{~B})$; this is comparable with humans $(19,25)$. The human thymus organoid also exhibits macrophage and B cell (medullary B cells) reconstitution restricted to the medulla (Figure 3B), which is comparable with human thymus $(19,26)$. Human spleen organoid in the BLTS humanized mice exhibits robust macrophage reconstitution, with macrophages cell (human $\mathrm{CD}^{+} 8^{+}$cells) levels highest in the red pulp and lowest in the white pulp (Figure 3B); this is comparable with healthy human spleen $(18,20)$. The human spleen organoid also exhibits human $\mathrm{T}$ and $\mathrm{B}$ cells reconstitution, which is overwhelmingly restricted to the white pulp, with human $\mathrm{T}$ and $\mathrm{B}$ cells occupying distinct zones (Figure $3 \mathrm{~B}$ ), as exhibited in human spleen (18).

Human immune cells in the BLTS humanized mice also reconstitute immunodeficient mouse lymphoid organs, specifically spleen and lymph nodes; however, said humanized murine spleen and humanized murine lymph node differs from human lymphoid tissues in both microanatomy and immune cell distribution profile (Supplemental Figure 3) $(18,27)$. The humanized murine lymph nodes in the BLTS humanized mice exhibit human macrophages, $\mathrm{T}$ and $\mathrm{B}$ cell reconstitution; however, lymphoid tissue structures associated with optimal human lymph node development, including paracortex and medulla, are absent (Supplemental Figure 3, A and B) (27). The humanized murine spleen exhibits human $\mathrm{T}$ and $\mathrm{B}$ cell reconstitution in the BLTS humanized mice, but with less distinct organization of $\mathrm{T}$ and $\mathrm{B}$ cell zones; red pulp development and human macrophage (human CD68 ${ }^{+}$ cells) reconstitution is poor compared with human spleen (Supplemental Figure 3, C and D) (28). The suboptimal development of humanized murine lymphoid tissues in the BLTS humanized mice is comparable with the suboptimal development of humanized murine lymphoid tissue structures in the BLT humanized mice (Supplemental Figure 4A) (29). 

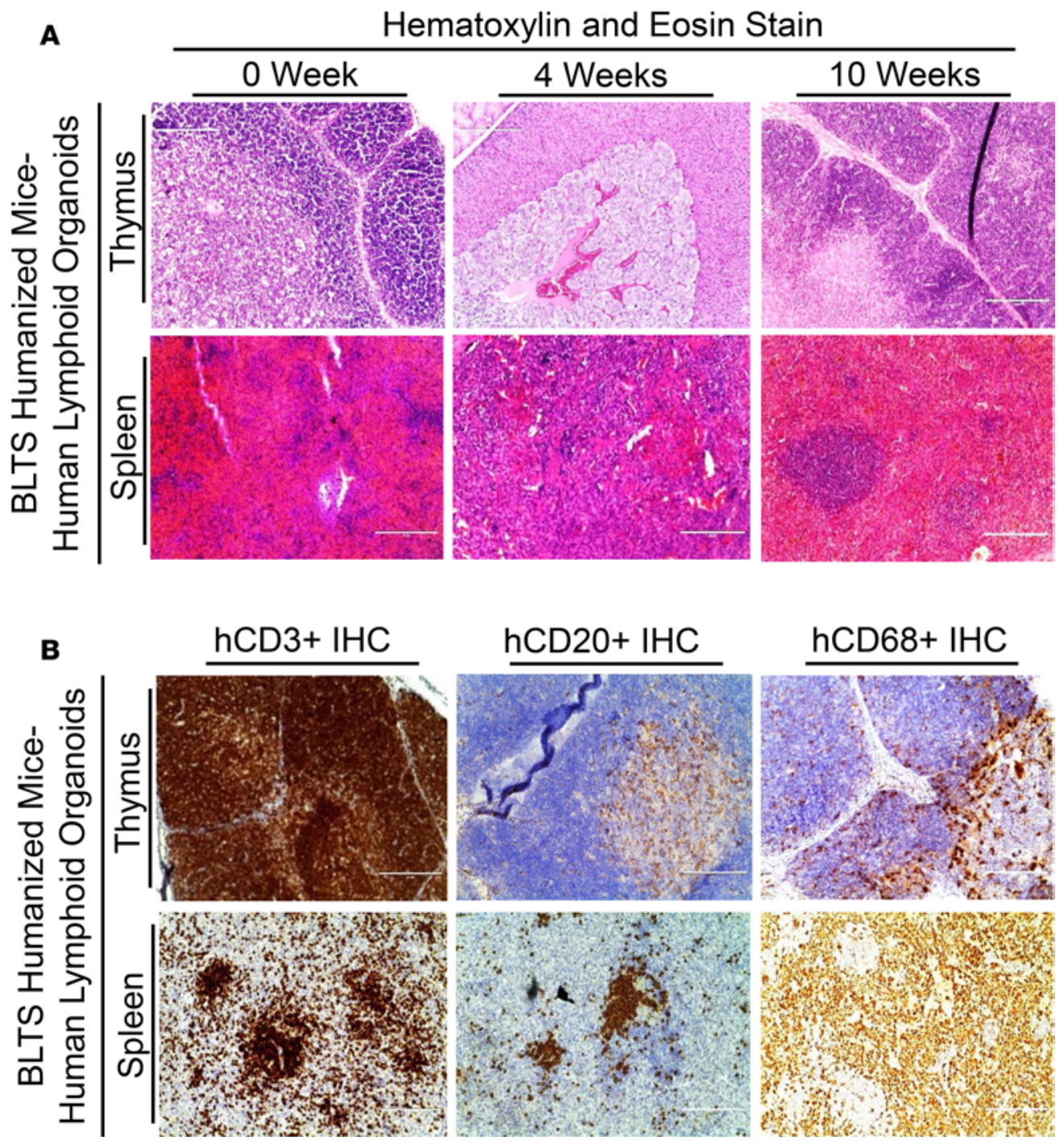

Figure 3. Human immune cells development in lymphoid tissues in the BLTS humanized mouse model. (A) Representative histological (H\&E stain) analysis of human spleen and thymus organoids in BLTS humanized mice ( $n=4$ per group) at indicated time points after transplantation. (B) Representative human-specific immunohistochemical (Brown stain; T cells, hCD3+; B Cells, hCD20+; or macrophages-hCD68+) analysis of human spleen and thymus organoids in BLTS humanized mice ( $n=4$ per group) at 10 weeks after transplantation. Scale bars: $200 \mu \mathrm{m}$.

The blood is a major component of the immune system; it is composed of various lineages of immune cells, which play a critical role in eradicating or controlling of infectious agents, including HIV. It is well established that human immune cells reconstitute the peripheral blood of human immune system-humanized mouse models, including the BLT humanized mouse model (Supplemental Figure 5). We examined human immune cell reconstitution and the dominant immune cell subset within peripheral blood mononuclear cells (PBMCs), namely lymphocytes in BLTS humanized mice (Figure 4). Analysis of the PBMCs in BLTS humanized mice showed high $(\sim 78 \%)$ human immune cell (human $\mathrm{CD} 45^{+}$cells) reconstitution in the peripheral blood, with human $\mathrm{T}$ (human $\mathrm{CD}^{+}$) cells (including human $\mathrm{CD}^{+}$and $\mathrm{CD} 8^{+}$subsets at $\sim 1.5: 1$ ratio) and $\mathrm{B}$ (human $\mathrm{CD} 19^{+}$) cells (Figure $4, \mathrm{~A}$ and $\mathrm{B}$ ) reconstituted ( $77 \%$ of human PBMCs) at comparable levels to lymphocytes population in the peripheral blood of healthy humans (30-32). In addition to demonstrating robust human immune cell reconstitution in lymphoid tissues and the peripheral blood, we also showed that the BLTS humanized mouse model is reconstituted with human immune cell subtypes that are targets for HIV replication, namely CD4 ${ }^{+} \mathrm{T}$ cells and macrophages (Figure 3 and Figure 4).

The BLTS mouse model supports HIV viremia and ART-mediated suppression of HIV viremia. Humanized mouse models reconstituted with human immune cells and/or lymphoid tissues are the only models for in 

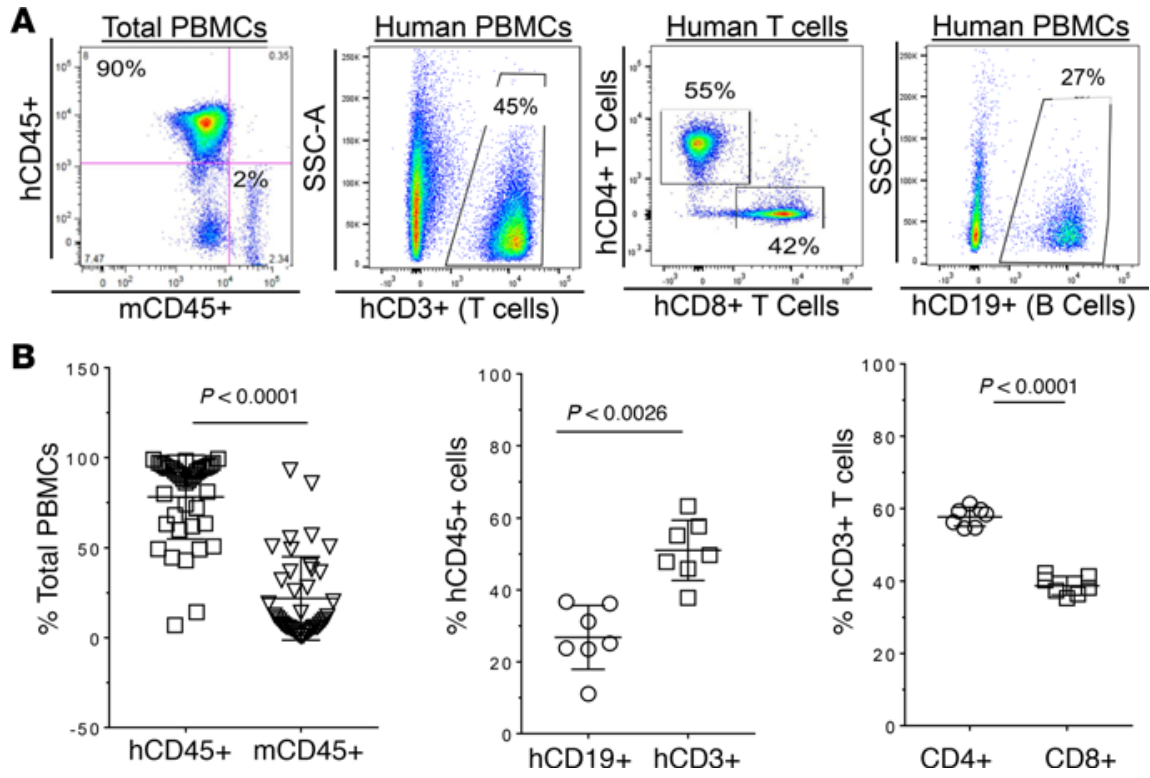

Figure 4. Human immune cell development in the peripheral blood in the BLTS humanized mouse model. (A) Representative flow cytometry analysis of human immune cell (hCD45') reconstitution, along with lymphocytes subsets, including T cells (CD3+) and $C D 4^{+}$and $C D 8^{+} T$ cell subsets and B cells (CD19+) in peripheral blood mononuclear cells (PBMCs) of BLTS humanized mice at 10 weeks after transplantation. (B) Quantification of human immune cells reconstitution ( $n=40$; 3 independent experiments) and lymphocyte subsets $(n=7)$ in PBMCs of BLTS humanized mice at 10 weeks after transplantation. Data is presented as mean values \pm SD. $P$ values were determined using paired, 2 -tailed Student's $t$ test between 2 groups.

vivo mechanistic studies of HIV infection, pathogenesis, and human immune response (33). To investigate HIV replication dynamics in the context of an in vivo "human immune system"model, we infected BLTS humanized mice with HIV. HIV replication kinetics in the BLTS humanized mice (Figure 5A) exhibits similarity to HIV replication kinetics in adult humans (34), with viral load peaking at 2 weeks after infection and subsequently dropping by $\sim 1.5 \mathrm{log}$, beginning at 6 weeks after infection, to a stable set-point at 12 weeks after infection (Figure 5A). For comparative analysis of HIV replication kinetics in BLTS and BLT humanized mice, we used mice transplanted at the same time, using cells and tissues from the same donor and under the same conditions and experimenter, with the only exception being the inclusion or exclusion of the human spleen organoid. Additionally, HIV infection of BLTS and BLT humanized mice was performed under the same conditions, using the same dose of inoculum and by the same experimenter. Comparative analysis of HIV replication kinetics in BLT and BLTS humanized mice shows the absence of significant change in viremia levels in BLT humanized mice after initial peak at 2 weeks after infection; however the viral set point is similar between both models (Figure 5A and Supplemental Figure 6). The lack of significant change in viral load in the BLT humanized mice after 2 weeks after infection is consistent with published reports $(35,36)$. Mock-inoculated and HIV-infected BLT humanized mice were euthanized at 12 weeks after infection and 24 weeks after transplantation for humane reasons due to severe GVHD, while BLTS mice were extended to 24 weeks after infection and 36 weeks after transplantation.

The global current standard of care for HIV infection is daily administration of ART, which reduces viral load to approximately $<50$ copies per $\mathrm{ml}(37,38)$; however, HIV persists in reservoir, which are reactivated within approximately 4 weeks upon cessation of therapy (39). We administered ART daily for 4 weeks to HIV-infected BLTS humanized mice beginning at 8 weeks after infection and showed that ART significantly reduces ( $>1.5 \log$ reduction) HIV viral load within 1 week of treatment, with reduced HIV viral load maintained over the duration of the 4-week treatment (Figure 5B). Withdrawal of ART in viral load-suppressed chronic HIV-infected BLTS humanized mice resulted in viral load rebound within 2 weeks, with viral load levels reaching set point levels within 8 weeks after ART withdrawal (Figure 5B). For analysis of replication-competent HIV in the BLTS humanized mouse model, we utilize the TZM-bl cell line, which stably expresses CD4, CCR5, and CXCR4 and carries an integrated copy of the $\beta$-galactosidase ( $\beta$-gal) gene under control of an HIV-1 long terminal repeat (LTR) promoter, to quantify inducible replication competent HIV-1 
A

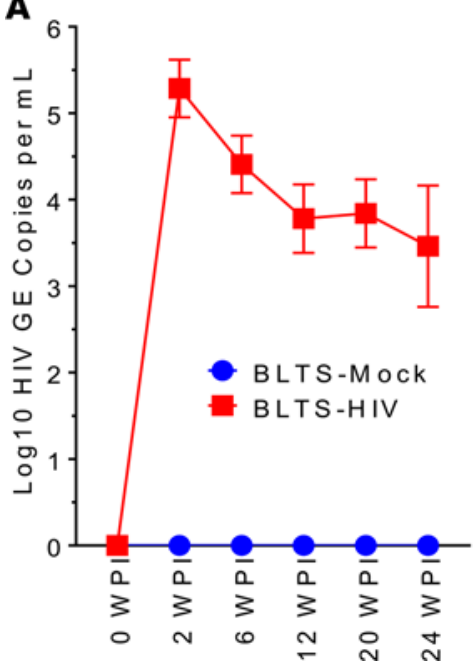

B

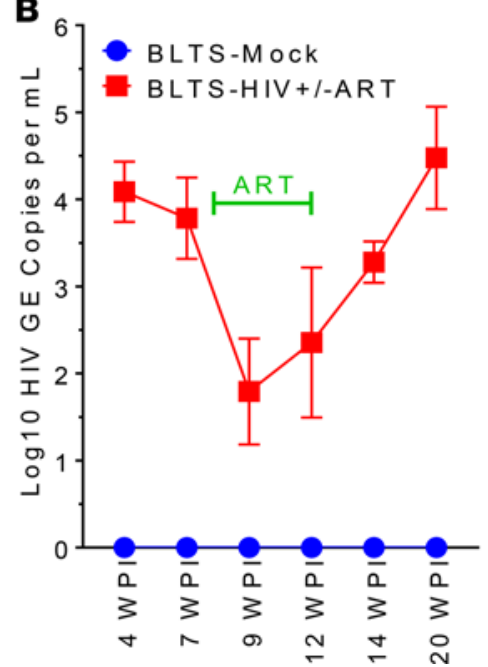

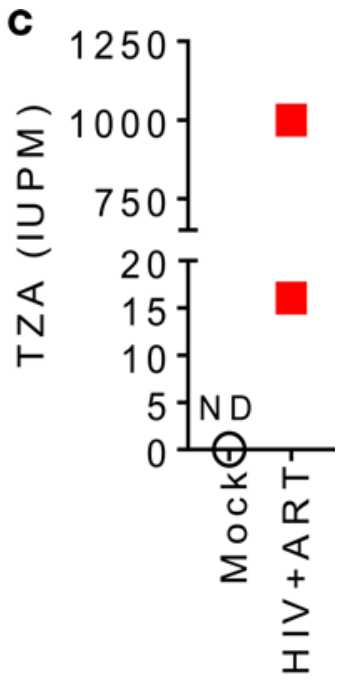

Figure 5. HIV replication kinetics in the BLTS humanized mouse model. (A) Kinetics of HIV-1 replication (HIV RNA genome copies per ml, HIV GE copies per $\mathrm{ml}$ ) in the blood following inoculation at $1 \times 10^{5}$ IU per mouse in BLTS humanized mice ( $n=4$ per group). (B) Kinetics of HIV-1 replication (HIV RNA genome copies per mI, HIV GE copies per ml) in the blood of chronic HIV-infected BLTS humanized mice following intervention with triple antiretroviral drug therapy (ART) ( $n=6$ per group) and subsequent cessation after 4 weeks of treatment ( $n=3$ per group); viral load was allowed to rebound over a period of 8 weeks. (C) Chronic HIV infection in BLTS humanized mice was allowed to develop over a period of 8 weeks, and animals were subsequently treated with ART for 4 weeks, at which point replication-competent HIV in human resting CD4 ${ }^{+}$T cells from the human spleen organoid of individual mice were analyzed $(n=2)$ using the TZA method, with CD4 ${ }^{+} T$ cells activated using CD3/CD28 stimulation; age-matched mock-inoculated BLTS humanized mouse served as control (ND abbreviates not detectable). Data is presented as mean values \pm SEM.

in an assay, termed TZA $(40,41)$. We demonstrated the presence of replication-competent HIV in human spleen organoid-derived human resting $\mathrm{CD} 4^{+} \mathrm{T}$ cells $\left(\sim 5 \%\left[\sim 2.5 \times 10^{6}\right.\right.$ to $\left.4 \times 10^{6}\right]$ of total splenocytes $[\sim 50$ $\times 10^{6}$ to $\left.\left.80 \times 10^{6}\right]\right)$ from ART-treated, HIV-infected BLTS humanized mice (Figure $5 \mathrm{C}$ ). The detection of replication-competent HIV in the human spleen in ART-treated, HIV-infected BLTS humanized mice is consistent with studies demonstrating that the human spleen is a HIV sanctuary site during ART in humans (42).

Chronic HIV infection in BLTS humanized mice induces progressive tissue fibrosis in human lymphoid tissues. Several studies have demonstrated that chronic HIV infection results in progressive lymphoid tissue fibrosis, which is not completely eradicated by long-term ART (43-45). Therefore, we examined chronic HIVinduced lymphoid tissue fibrosis in the BLTS humanized mouse model. Chronic HIV infection results in progressive collagen deposition (tissue fibrosis) in human lymphoid tissues (human spleen and thymus organoids) in the BLTS humanized mouse model as measured using Sirius red/fast green stain (Figure 6A). We also confirmed HIV-induced collagen deposition in human lymphoid tissues (human spleen and thymus organoids) in BLTS humanized mice via Masson's trichrome stain and demonstrated that the excessive collagen deposition is of human origin via IHC detecting human collagen 1 (Figure 6B). We also examined lymphoid tissue fibrosis in ART-treated, chronic HIV-infected BLTS humanized mice; significantly higher levels of collagen were detected in human lymphoid tissues (human spleen and thymus organoids) from ART-treated (4 weeks), chronic HIV-infected BLTS humanized mice at 12 weeks after infection compared with mock-inoculated mice (Figure 6C).

Chronic HIV infection in BLTS humanized mice also induces progressive collagen deposition in the humanized murine spleen, albeit at a significantly slower rate and lower level, as compared with the human spleen organoid (Figure 6 and Supplemental Figure 7, A and B). Excessive collagen or human collagen 1 is not detectable at 10 weeks after HIV infection in the humanized murine spleen, and the level of collagen in the humanized murine spleen at 24 weeks after HIV infection $\left(\sim 7.6 \%\right.$ of tissue area Sirius red $\left.{ }^{+}\right)$is lower compared with the human spleen organoid $\left(\sim 28.8 \%\right.$ of tissue area Sirius red $\left.{ }^{+}\right)$at 24 weeks after HIV infection (Supplemental Figure 7, A and B, and Figure 6A). ART-treated (4 weeks), chronic HIV-infected BLTS humanized mice at 12 weeks after infection exhibited significantly higher levels of collagen deposition in humanized murine lymph nodes compared with mock-inoculated mice (Supplemental Figure 7C). For comparative analysis, we examined collagen deposition in another human immune system-humanized 


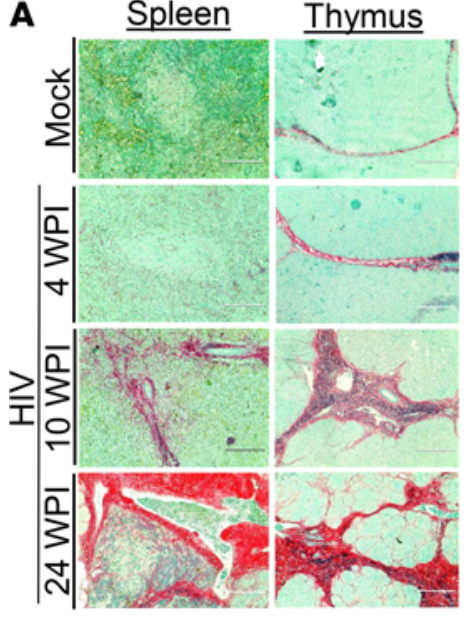

B

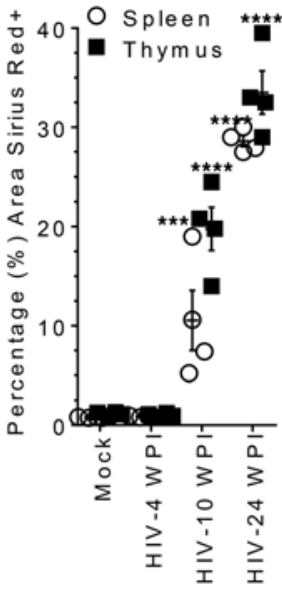

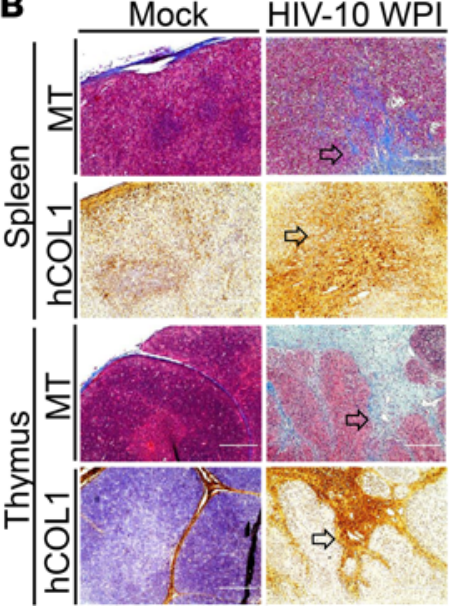

C

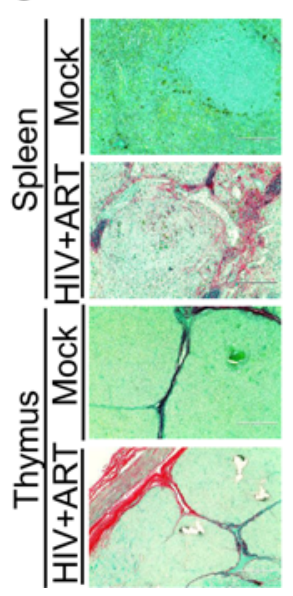

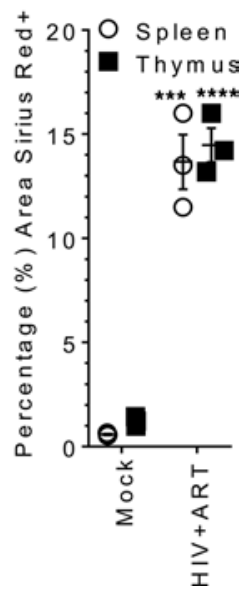

Figure 6. HIV infection induces progressive tissue fibrosis in human lymphoid tissues in BLTS humanized mice. (A) Kinetics of lymphoid tissue fibrosis (Sirius red/fast green stain; collagen-positive, red; and collagen negative, green) in human spleen and thymus organoids ( $n=4$ per group) following inoculation at $1 \times 10^{5} \mathrm{IU}$ per mouse in BLTS humanized mice; mock inoculated mice; and age-matched mice to 24 weeks after infection (24 WPI) served as controls. (B) Lymphoid tissue fibrosis analysis was confirmed independently via Masson's trichrome stain (MT; aniline blue, collagen positive; dark red/purple, cell nuclei; and red/pink, cell cytoplasm) and human collagen 1 (hCOL1) IHC in human spleen and thymus tissues in HIV-infected BLTS humanized mice at 10 WPI. Arrows indicate same region of the tissue across the different staining. (C) Analysis of lymphoid tissue fibrosis in human spleen and thymus organoids of 4-week ART-treated HIV-infected BLTS humanized mice. Chronic HIV infection in BLTS humanized mice was allowed to develop over a period of 8 weeks, and animals were subsequently treated with ART for 4 weeks, at which point lymphoid tissues ( $n=3$ per group) were analyzed for collagen deposition using Sirius red/fast green stain (red, collagen-positive; green, collagen-negative); age-matched mice served as controls. Scale bars: $200 \mu \mathrm{m}$. Data is presented as mean values \pm SEM. $P$ values ( ${ }^{* *} P<0.001,{ }^{* * *} P<0.0001$ ) were determined using 1 -way ANOVA between more than 2 groups or unpaired, 2 -tailed Student's $t$ test between 2 groups for each lymphoid tissue, with mock as the control group.

mouse model, namely the BLT humanized mouse model. Analysis of collagen deposition (fibrosis) in the human thymus organoid and humanized murine spleen and lymph node tissues in BLT humanized mice via Sirius red/fast green staining showed marginal levels of fibrosis in the human thymus and the humanized murine lymph nodes and showed the absence of fibrosis in the humanized murine spleen at 12 weeks after HIV infection compared with mock-inoculated mice (Supplemental Figure 8).

Chronic HIV infection-induced lymphoid fibrosis in BLTS humanized mice is associated with immunodeficiency. Several reports have demonstrated that chronic HIV-associated lymphoid tissue fibrosis is associated with $\mathrm{CD}^{+} \mathrm{T}$ cell depletion in humans $(44,45)$. Additionally, the levels of $\mathrm{CD}^{+} \mathrm{T}$ cell reconstitution following intervention with ART is strongly associated with the levels of existing lymphoid fibrosis $(43,45)$. We examined the relationship between human immune cell depletion and fibrosis in human lymphoid tissues. First, immunohistochemical analysis of lymphoid follicles in the human spleen organoid showed that chronic HIV infection progressively depletes both human $\mathrm{T}$ and $\mathrm{B}$ cells in their respective zones in the follicles (Figure 7A). Second, immunohistochemical analysis of the dominant immune cell type for each lymphoid tissue (macrophages $\left[\mathrm{CD}^{+} 8^{+}\right]$cells in the spleen and $\mathrm{CD}^{+} \mathrm{T}$ cells in the thymus) showed chronic HIV infection progressively depletes both macrophages and $\mathrm{CD}^{+} \mathrm{T}$ cells in human lymphoid tissues (Figure 7B). Furthermore, we demonstrated HIV replication in the human spleen organoid, which was associated with macrophage (CD163 $\mathrm{RNA}^{+}$cells) depletion (Figure 7C). Correlation analysis of immunodeficiency in lymphoid tissues and lymphoid tissue fibrosis showed that human macrophage depletion in the human spleen organoid and human $\mathrm{CD}^{+} \mathrm{T}$ cell depletion in the human thymus organoid strongly correlates with collagen deposition in human lymphoid tissues in HIV-infected BLTS humanized mice (Figure 7D).

\section{Discussion}

Lymphoid tissue fibrosis is a major pathogenic feature associated with chronic HIV infection with or without ART; however, the mechanisms of HIV-induced lymphoid tissue fibrosis remains to be fully elucidated $(7,8)$. Several reports suggest a nexus between lymphoid tissue fibrosis, chronic inflammation, immunodeficiency, and immune impairment in chronic HIV infection $(45,46)$. The role of HIV-induced lymphoid tissue fibrosis in persistent immune abnormalities, including limited immune reconstitution, chronic inflammation, and 
A

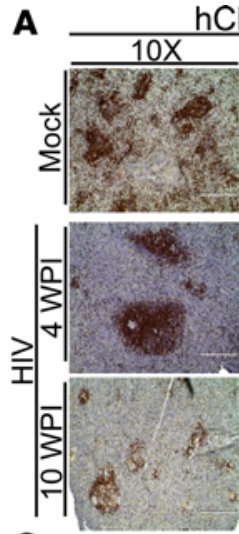

C
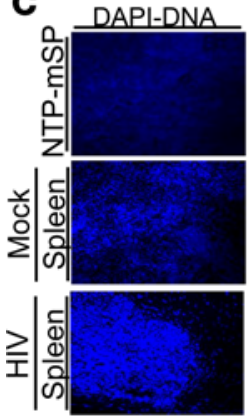
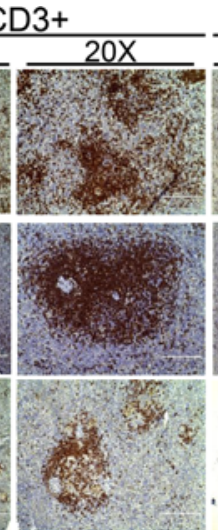

FITC-hCD163

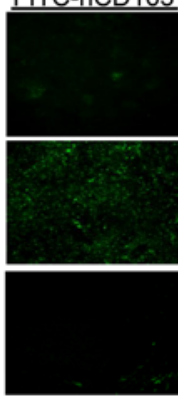

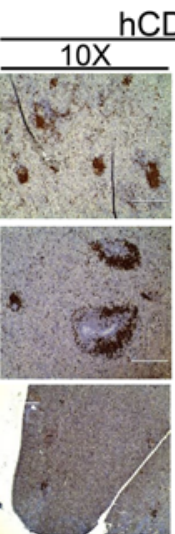

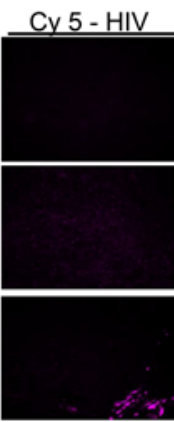

$\mathrm{CD} 20+$

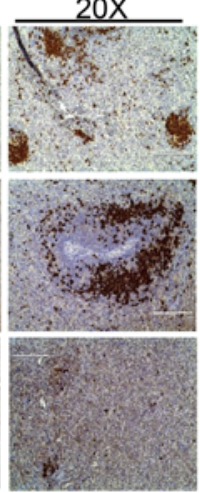

Merged

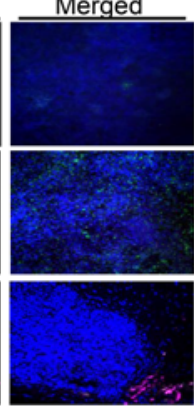

B
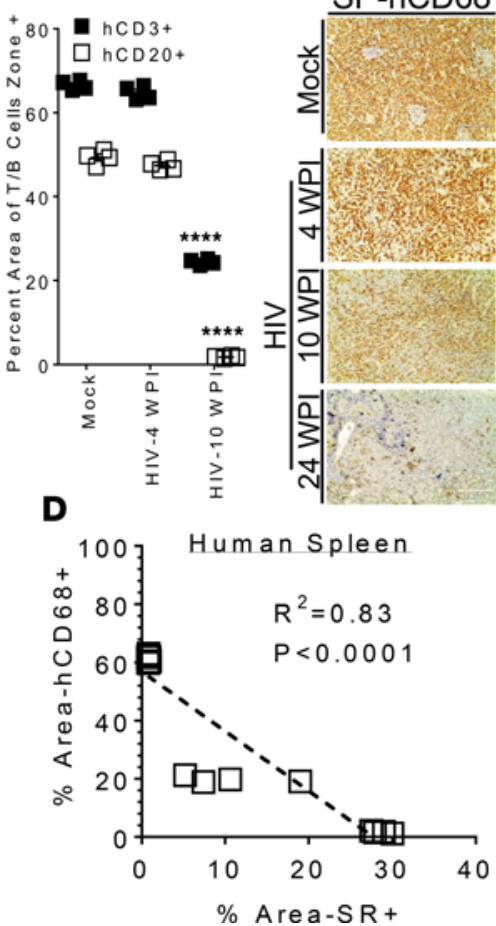

B SP-hCD68 Thy-hCD4

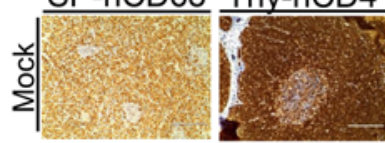

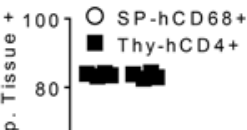
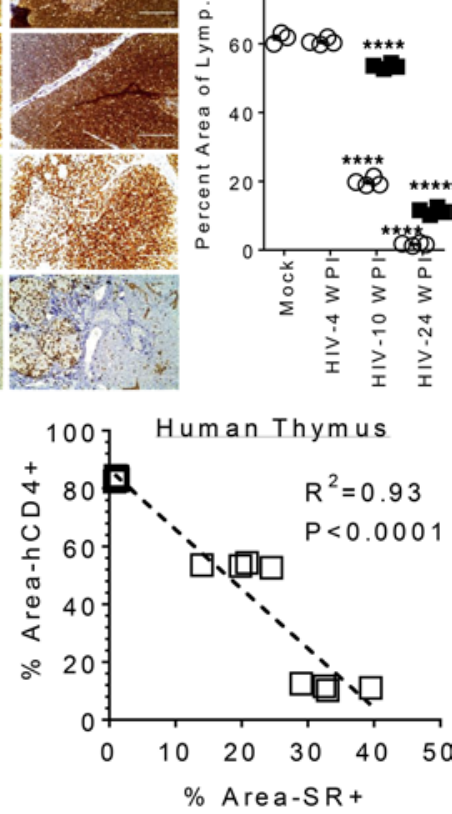

Figure 7. HIV-induced immunodeficiency correlates with lymphoid tissue fibrosis in the BLTS humanized mouse model. (A and B) Human-specific immunohistochemical analysis of the kinetics of immunodeficiency in human lymphoid tissues, (A) T (Brown stain, hCD3+) and B (Brown stain, hCD20 $)$

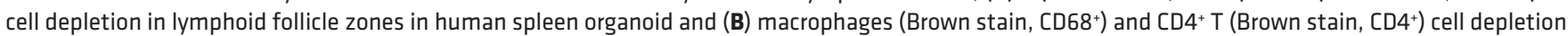
in human spleen (SP) and thymus (Thy) organoids, respectively, following inoculation at $1 \times 10^{5}$ IU per mouse in BLTS humanized mice; mock inoculated mice, age-matched to the 24 weeks post-infection ( $24 \mathrm{WPI}$ ) served as controls. Immunohistochemical staining of spleens from nontransplanted NSC mice with the human antibodies were nonreactive (data not shown). (C) In situ hybridization (RNA expression) analysis of macrophage (CD163+, FITC, green fluorescence) levels and the presence of HIV viral RNA (red/pink fluorescence) in the human spleen organoid in mock-inoculated and chronic HIV-infected (24 weeks after infection) BLTS humanized mice; spleen from nontransplanted NSG mice (NTP-mSP) served as control. (D) Correlation analysis of human mac-

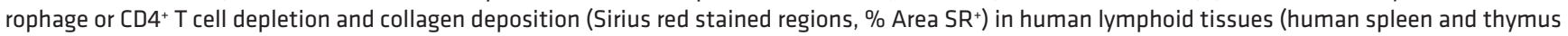
organoids). Representative images are shown ( $n=4$ per group), and data is presented as mean values \pm SEM. $P$ values (**** $P<0.0001)$ were determined using 1-way ANOVA between more than 2 groups for each immune cell staining or lymphoid tissue, with mock as the control group. R2 coefficient was determined using linear regression and 16 mice. Scale bars: $200 \mu \mathrm{m}$.

functional exhaustion of antiviral T cells in ART-suppressed individuals, remains to be clearly defined (43, 46). Additionally, the impact of HIV-associated lymphoid tissues fibrosis on the generation, trafficking, and function of anti-HIV immune cells (including T cells) within lymphoid tissues and associated follicles also remains to be clearly defined (46).

Understanding the mechanism of HIV-induced lymphoid tissue fibrosis could have a significant impact on the development of novel therapeutic strategies for curing HIV infection and treating persistent immune abnormalities associated with non-AIDS pathologies (38). Additionally, lymphoid tissue fibrosis could have a significant impact on T cells/therapeutic vaccine-based HIV cure strategies, as recent studies demonstrate that lymphoid tissues, including lymph nodes (47) and spleen (42), are sanctuary organs for HIV. The stromal network of lymphoid tissues, which are damaged by fibrosis, play a critical role in the trafficking and functionality of $\mathrm{T}$ cells (7). The in vivo functionality of infused anti-HIV $\mathrm{T}$ cells or the induction of robust anti-HIV immune response via therapeutic vaccine in HIV-induced fibrotic lymphoid tissues are unexplored areas due in part to the lack of robust small animal models. Developing a small animal model that recapitulates chronic HIV infection and associated lymphoid tissue pathologies and immune abnormalities is critical for addressing the above discussed unexplored areas (38).

We established a human immune system-humanized mouse model, termed BLTS humanized mouse model, that includes autologous human spleen and thymus organoids and human immune cells in order to develop a small animal model of chronic HIV infection-induced fibrosis in human lymphoid tissues. The BLTS humanized mouse model supports robust human immune cell development, along with robust development of 
lymphoid organs, including human thymus and spleen, which were comparable with human lymphoid organs. The BLTS humanized mouse model addresses the problem of suboptimal macrophage reconstitution (29) and poor lymphoid tissue architecture (13) in the humanized murine spleen of human immune system-humanized mouse models via incorporation of a human spleen organoid. The BLTS humanized mouse model supports robust human immune cell reconstitution (including macrophages) and lymphoid tissue microanatomy in the human spleen organoid, with immune cell types and their relative levels and spatial distribution comparable with human spleen (18). Most notably, the human spleen organoid supports robust red pulp macrophage development at comparable levels with human spleen (18). Several factors could account for the robust human macrophage reconstitution observed in the human spleen organoid in BLTS humanized mice. Firstly, the human spleen-stromal network could facilitate optimal development of human tissue macrophages (48). Secondly, optimal development of human tissue macrophages in the spleen is not mediated by hematopoietic stem cells but, instead, by embryonic-derived resident spleen macrophages, which are transplanted with the human spleen organoid (48). The BLTS humanized mouse model also supports robust human immune cell reconstitution and lymphoid tissue architecture in the human thymus organoid, with immune cell types (T cells, macrophages, and B cells) and their relative levels and spatial distribution comparable with human thymus (19).

The BLTS humanized mouse model also supports reconstitution of immunodeficient mouse lymphoid organs with human immune cells, resulting in the development of humanized murine spleen and lymph nodes (axillary and mesenteric lymph nodes), albeit the humanized murine lymphoid tissue development is suboptimal compared with human lymphoid tissues. The inclusion of the human spleen organoid could have a significant impact on the overall human immune system development and associated immune response to infectious agents (including HIV) in human immune system-humanized mice, as the spleen is a major secondary lymphoid organ, with critical roles in the development of antigen-specific $\mathrm{T}$ and $\mathrm{B}$ cells, and is a major deposit of antigen presenting cells and the mononuclear phagocyte system (49); future studies should address those areas. Interestingly, extremely low $(<10 \%)$ incidence of GVHD was observed in BLTS humanized mouse model as high as 9 months after transplantation and near the natural life span of the NSG mice ( $~ 12$ months), with instances $(<10 \%)$ of GVHD associated with the failure of codevelopment of human spleen organoid (i.e., the absence of the spleen graft) and the human thymus organoid. In contrast, BLT humanized mice predictively exhibit severe GVHD (fur loss and wasting syndrome) by 6 months after transplantation (21) in comparative studies using the same procedures and donor tissues/cells across both BLTS and BLT humanized mice. Several factors contribute to the development of GVHD, including HLA class I alleles; future studies should rigorously examine GVHD across BLT and BLTS humanized mice using the donor tissues with HLA class I alleles of high and low susceptibility for GVHD (21). Furthermore, the impact of the human spleen organoid and associated resident immune cells (i.e., myeloid cells) on the development of chronic GVHD should also be investigated. A possible mechanism of the reduced GVHD in the BLTS humanized mouse model could be the engraftment/development of tolerogenic immune cells in the human spleen, namely tolerogenic DCs and Tregs, which plays a major role in modulating immune cell (i.e., T cell) activation (28). Future studies should examine the development of DCs and Tregs within the human spleen organoid and other lymphoid tissues in the BLTS humanized mouse model.

To develop a small animal model for HIV-induced lymphoid fibrosis, we infected BLTS humanized mice with HIV. Interestingly, HIV replication kinetics in BLTS humanized mice is comparable with adult humans (34) but differs from BLT humanized mice. BLTS humanized mice exhibit a relatively higher peak viral load, which subsequently drops to a lower viral set point and remains stable. Several factors could account for the viral load kinetics in BLTS humanized mice. First, increase in the number of cells susceptible to HIV infection (i.e., spleen macrophages) could account for the higher peak viral load, and subsequent reduction to a lower set point could result from anti-HIV immune response. Alternatively, the presence of cells that support robust replication but undergo rapid depletion without subsequent reconstitution or impaired immune reconstitution could account for the viral load kinetics in BLTS humanized mice. Future studies should rigorously examine the contribution of the various lymphoid tissues to the viremia and the human immune response associated with infection over time. Future studies should also rigorously examine HIV cellular reservoirs and their localization within human lymphoid tissues in BLTS humanized mice. Histochemical examination of lymphoid tissues demonstrated that chronic HIV infection induces progressive tissue fibrosis in both primary and secondary human lymphoid tissues in the BLTS humanized mouse model, which persists despite ART (36), albeit with a short-term course of treatment. The HIV-induced lymphoid tissue fibrosis correlated with macrophages and $\mathrm{CD} 4^{+} \mathrm{T}$ cells loss in human lymphoid tissues in 
the BLTS humanized mouse model. Additionally, HIV infection in the BLTS humanized mice also results in progressive loss of lymphoid follicles; thus, HIV-associated immunopathogenesis also negatively impacts the functionality of lymphoid tissues. These findings are consistent with clinical studies in humans demonstrating that HIV infection induces progressive lymphoid tissues fibrosis, which is not eradicated by ART and is associated with suboptimal immune reconstitution $(5,8)$. Future studies should include an early-/ long-term course of treatment to determine the impact of ART on lymphoid tissue fibrosis progression/ regression in chronic HIV infection.

Several lines of evidence suggest that stromal cells, including fibroblasts, play a critical role in modulating tissue architecture and excessive collagen deposition (tissue fibrosis) (50). Interestingly, HIV infection induced limited fibrosis in humanized murine lymphoid tissues compared with human lymphoid tissues in the BLTS humanized model, suggesting human lymphoid tissue stromal cell and HIV/HIV-infected cell interactions could play a critical role in HIV-induced lymphoid fibrosis. Additionally, the HIV-induced collagen in human lymphoid tissues in the BLTS humanized model is of human origin, thus demonstrating a human-mediated lymphoid tissue fibrosis. Myeloid cells such as macrophages are resident cells in a myriad of tissues, including lymphoid tissues (50). It is well established that macrophages interact with stromal cells and play a critical role in tissue repair, remolding, and fibrosis (50). We demonstrated that HIV infection induced lymphoid fibrosis in the BLTS humanized mouse model, which exhibits robust macrophage reconstitution, while the BLT humanized mouse, which exhibits poor macrophage reconstitution, exhibited marginal lymphoid tissue fibrosis following HIV infection.

In summary, we report the development of a potentially novel human immune system-humanized mouse model that incorporates human spleen and thymus organoids, along with human immune cells, termed BLTS humanized mouse model. The BLTS humanized mouse model addressed several limitations associated with human immune system-humanized mouse models, including development of GVHD and suboptimal development of the mononuclear phagocyte system (i.e., macrophages). The BLTS humanized mouse model provides a robust small animal model for in vivo mechanistic studies of HIV-induced fibrosis in human lymphoid tissues and the impact of lymphoid fibrosis on HIV pathogenesis and HIV cure strategies. The BLTS humanized mouse model also provides a potentially novel platform for mechanistic studies of the interactions between stromal cells and immune cells in HIV-induced fibrosis in human lymphoid tissues, as well as in vivo evaluation of therapeutics targeting HIV-induced lymphoid tissue fibrosis. Importantly, the BLTS humanized mouse model could enable the unraveling of the nexus between lymphoid tissue fibrosis and persistent immune abnormalities, including chronic inflammation, functional exhaustion of antiviral $\mathrm{T}$ cells, and limited immune reconstitution under conditions of robust ART-mediated HIV suppression. Additionally, human immune system-humanized mouse models have been employed for in vivo mechanistic studies for a myriad of human diseases, including cancer $(51,52)$, viral infections (53-62), and bacterial infections $(63,64)$; thus, the BLTS humanized mouse model represents a potentially novel in vivo platform for biomedical research.

\section{Methods}

Construction of human immune system-humanized mice. Male and female NOD.Cg-Prkdc ${ }^{\text {scid }} \mathrm{Il}^{2 \mathrm{rg}^{\mathrm{tm}} 1 \mathrm{w}_{\mathrm{jl}} / \mathrm{SzJ}}$ (NSG) mice were obtained from the Jackson Laboratory (stock no. 005557) and bred in the Division of Laboratory Animal Resources (DLAR) facility at the University of Pittsburgh. Human fetal tissues were obtained from the Health Sciences Tissue Bank at the University of Pittsburgh, and tissues were handled and processed under biosafety level 2 conditions. Male and female BLTS humanized mice and male and female BLT humanized mice were generated as previously reported, with the exception being the incorporation of human spleen organoids in the lymphoid tissue grafts in the BLTS mouse model $(29,35,36)$. In comparative studies between BLT and BLTS humanized mouse models, mice were transplanted with cell/tissues from the same donor under the same conditions by the same experimenter within a 12-hour period. Briefly, adult NSG mice were sublethally irradiated and anesthetized; $\sim 1-\mathrm{mm}^{2}$ fragments of autologous human fetal thymus and liver with or without spleen were implanted under the kidney capsule. CD $34^{+}$hematopoietic progenitor cells purified from fetal liver of the same donor were injected i.v. (via retroorbital route) following implantation of the lymphoid tissues. Mice were housed under specific pathogen free conditions and fed irradiated chow (Prolab Isopro RHM 3000 Irradiated, catalog 5P75-RHI-W 22, PMI Nutrition International) and autoclave water. Human immune cell engraftment was detected by flow cytometry at 10 weeks after transplantation of lymphoid tissues and autologous hematopoietic stem cells. 
Blood cell analysis. Peripheral blood cells were analyzed using flow cytometry. Briefly, peripheral blood was collected from humanized mice and mixed with $20 \mathrm{mM}$ EDTA at a 1:1 ratio; single cell leukocytes were prepared via red blood cells lysis using Ammonium-Chloride-Potassium (ACK) buffer. Single-cell suspensions prepared from peripheral blood of humanized mice were stained with a LIVE/DEAD Fixable Aqua Dead Cell Stain Kit (Thermo Fisher Scientific), fluorochrome-conjugated antibodies (anti-mouse CD45, BioLegend, catalog 103126; anti-human CD45, BioLegend, catalog 304014; anti-human CD3, BioLegend, catalog 300312; anti-human CD4, BioLegend, catalog 317410; anti-human CD8, BioLegend, catalog 300906; anti-human CD19, BioLegend, catalog 302232), fixed, and analyzed on a BD LSRFortessa cell analyzer - flow cytometer (BD Biosciences). Data were analyzed using FlowJo software. Briefly, leukocytes were selected based on forward and side scatter. Single cell and live leukocytes were selected for further analysis of the percentage of human leukocytes (anti-human CD45, $\mathrm{hCD} 45^{+}$) and mouse leukocytes (anti-mouse $\mathrm{CD} 45^{+}, \mathrm{mCD} 45^{+}$) in the peripheral blood. Subsequent analysis of the various human lymphocyte populations and subsets were gated on human leukocytes.

Gross/tissue analysis. Gross analysis was performed using a camera (8 mega pixel); animals were either euthanized or anesthetized prior to photographing. Indicated tissue samples from humanized mice, BALB/cJ mice (the Jackson Laboratory; stock no. 000651; 5 months old) or humans (thymus, Carolina Biological Supply Company, catalog 314388; spleen, BioChain, catalog T2234246; tissues were projected to be juvenile/adult tissues based microanatomy and/or supplier's designation; refs. 65-68) were fixed with formalin and subsequently embedded in paraffin. Paraffin-embedded fixed sections were stained with H\&E, Sirius red/fast green (collagen/fibrosis; Polysciences Inc., catalog 24901-250), or Masson's trichrome Stain Kit (collagen/fibrosis; Polysciences Inc., catalog 25088-100) and validated in situ hybridization RNA probes (Advanced Cell Diagnostics, anti-human CD163, catalog 417061; anti-HIV1 CladeB, catalog 425531) or with indicated anti-human antibodies (anti-human CD3, Biocare Medical, catalog CME 324 A, B, C; anti-human CD68, Biocare Medical, catalog CM 033A, -B, -C; anti-human CD20, Biocare Medical, catalog ACR 3004A, -B; anti-human collagen 1, Abcam, catalog ab138492). Reactivity of in situ hybridization RNA probes was determined using RNAscope Multiplex Fluorescent Assay (Advanced Cell Diagnostics). Immunoreactivity of indicated antibodies was determined by incubation with DAB substrate (MACH 2 Detection Kit, Biocare Medical) and counterstained with hematoxylin. Quantification of Sirius red or immunoreactivity of indicated anitibodies was performed using the ImageJ software (NIH) and following developer recommended methods for quantification.

HIV infection of human immune system-humanized mice. The CCR5-tropic strain of HIV-1 (NL4 with the CCR5-tropic BaL envelope) (69) was generated by transfection of 293T cells (ATCC; ATCC CRL-3216) with plasmid containing full-length HIV-1 genome, thus generating 293T supernatant with HIV; virus was titered on GHOST cells (NIH AIDS Reagent Program; catalog 3942) (70). 293T supernatant without HIV was used as mock control. Humanized mice with stable human leukocyte reconstitution were anesthetized and inoculated with mock or HIV-1 ( $\sim 1 \times 10^{5}$ infectious units) i.v. (via retroorbital route) or via intravaginal route.

ART in HIV-infected human immune system-humanized mice. Chronic HIV-infected humanized mice were treated with ART (daily i.p. injections emtricitabine [FTC, Cayman Chemicals, catalog 16879, $200 \mathrm{mg}$ / $\mathrm{kg}$ body weight], tenofovir disoproxil fumarate [TDF, Cayman Chemicals, catalog 16922, $200 \mathrm{mg} / \mathrm{kg}$ body weight], and raltegravir [RAL, Cayman Chemicals, catalog 16071, $80 \mathrm{mg} / \mathrm{kg}$ body weight) (36) beginning at 8 weeks after infection for a duration of 4 weeks. Viral load was allowed to rebound for a period of 8 weeks.

HIV-1 genomic RNA detection. Total RNA was purified from plasma using RNA-Bee (AMSBIO). The RNA was then reverse transcribed using TaqMan Reverse Transcription Reagents (Invitrogen) and quantitatively detected by real-time PCR using the TaqMan Universal PCR Master Mix (Invitrogen) with primers (forward primer, 5' - CCCATGTTTTCAGCATTATCAGAA - 3', and reverse primer, 5' - CCACTGTGTTTAGCATGGTGTTTAA - 3') and detection probe targeting HIV Gag gene (5' - AGCCACCCCACAAGA - 3') (71). The assay sensitivity/cutoff is 10 copies/ml (71).

$H I V-1$ replication competent reservoir in resting $C D 4^{+} T$ cells. Human splenocytes from human spleen organoids of individual mock or ART-treated chronic HIV-1 infected humanized mice with undetectable viral load ( $<10$ copies per $\mathrm{ml}$ ) were used in the TZA method $(40,72)$. Human resting CD4 ${ }^{+} \mathrm{T}$ cells were stimulated with CD3/28 to reactivate the HIV reservoir, and infectious units per million cells (IUPM) was determined following previously published methods.

Statistics. Significance levels of data were determined by using Prism7 (GraphPad Software). Experiments were analyzed by 2-tailed Student's $t$ test. Correlations between variables were evaluated using linear regression. A $P$ value less than 0.05 was considered significant. The number of animals are specified in each figure legend. 
Study approval. Human fetal liver and thymus (gestational age of 18-20 weeks) were obtained from medically or elective indicated termination of pregnancy through Magee-Women's Hospital of UPMC via the University of Pittsburgh, Health Sciences Tissue Bank. Written informed consent of the maternal donors was obtained in all cases, under IRB of the University of Pittsburgh guidelines and federal/state regulations. The use of human fetal organs/cells to construct humanized mice was reviewed by the University of Pittsburgh's IRB office, which has determined that this submission does not constitute human subject research as defined under federal regulations (45 CFR 46.102[d or f] and 21 CFR 56.102[c], [e], and [1]). The use of human hematopoietic stem cells was reviewed and approved by the Human Stem Cell Research Oversight (hSCRO) at the University of Pittsburgh. The use of biological agents (e.g., HIV), recombinant DNA, and transgenic animals was reviewed and approved by the Institutional Biosafety Committee (IBC) at the University of Pittsburgh. All animal studies were approved by the IACUC at the University of Pittsburgh and were conducted following NIH guidelines for housing and care of laboratory animals.

\section{Author contributions}

$\mathrm{MB}$ and PG conceived and designed experiments in the study. JS, SK, ANS, AS, AE, AD, KM, BR, AZ, WA, MD, and MB performed experiments. MB, JS, AS, and PG analyzed and interpreted the data. MB, JS, KM, SK, and PG prepared the manuscript.

\section{Acknowledgments}

Virus inoculum was provided by Zandrea Ambrose, Department of Medicine, University of Pittsburgh. This project used the UPMC Hillman Cancer Center and Tissue and Research Pathology/Health Sciences Tissue Bank shared resource, which is supported in part by award P30CA047904. Tissues were procured and isolated by the Staff of the University of Pittsburgh, Health Sciences Tissue Bank under an approved IRB. This work was supported by the NIH (R56AI126995). Diagrams and experimental schemes were made via modification of images from Creative Commons.

Address correspondence to: Moses Turkle Bility, Graduate School of Public Health, University of Pittsburgh, 130 DeSoto Street, Pittsburgh, Pennsylvania, USA 15261. Phone: 412.383.8056; Email: mtbility@pitt.edu.

1. Haase AT. Population biology of HIV-1 infection: viral and CD4+ T cell demographics and dynamics in lymphatic tissues. Annu Rev Immunol. 1999;17:625-656.

2. Estes JD, Haase AT, Schacker TW. The role of collagen deposition in depleting CD4+ T cells and limiting reconstitution in HIV-1 and SIV infections through damage to the secondary lymphoid organ niche. Semin Immunol. 2008;20(3):181-186.

3. Diaz A, et al. Factors associated with collagen deposition in lymphoid tissue in long-term treated HIV-infected patients. AIDS 2010;24(13):2029-2039.

4. Schacker TW, et al. Collagen deposition in HIV-1 infected lymphatic tissues and T cell homeostasis. J Clin Invest. 2002;110(8):1133-1139.

5. Schacker TW, et al. Lymphatic tissue fibrosis is associated with reduced numbers of naive CD4+ T cells in human immunodeficiency virus type 1 infection. Clin Vaccine Immunol. 2006;13(5):556-560.

6. Schacker TW, et al. Amount of lymphatic tissue fibrosis in HIV infection predicts magnitude of HAART-associated change in peripheral CD4 cell count. AIDS. 2005;19(18):2169-2171.

7. Zeng M, et al. Cumulative mechanisms of lymphoid tissue fibrosis and T cell depletion in HIV-1 and SIV infections. J Clin Invest. 2011;121(3):998-1008.

8. Sanchez JL, et al. Lymphoid fibrosis occurs in long-term nonprogressors and persists with antiretroviral therapy but may be reversible with curative interventions. J Infect Dis. 2015;211(7):1068-1075.

9. Thornhill JP, Fidler S, Klenerman P, Frater J, Phetsouphanh C. The Role of CD4+ T Follicular Helper Cells in HIV Infection: From the Germinal Center to the Periphery. Front Immunol. 2017;8:46

10. Zeng M, Haase AT, Schacker TW. Lymphoid tissue structure and HIV-1 infection: life or death for T cells. Trends Immunol. 2012;33(6):306-314.

11. Policicchio BB, Pandrea I, Apetrei C. Animal Models for HIV Cure Research. Front Immunol. 2016;7:12.

12. Victor Garcia J. Humanized mice for HIV and AIDS research. Curr Opin Virol. 2016;19:56-64.

13. Martinez-Torres F, Nochi T, Wahl A, Garcia JV, Denton PW. Hypogammaglobulinemia in BLT humanized mice--an animal model of primary antibody deficiency. PLOS ONE. 2014;9(10):e108663.

14. Kooreman NG, et al. Alloimmune Responses of Humanized Mice to Human Pluripotent Stem Cell Therapeutics. Cell Rep. 2017;20(8):1978-1990

15. Shibata S, et al. SCID-bg mice as xenograft recipients. Lab Anim. 1997;31(2):163-168.

16. Aronovich A, et al. Correction of hemophilia as a proof of concept for treatment of monogenic diseases by fetal spleen transplantation. Proc Natl Acad Sci USA. 2006;103(50):19075-19080.

17. Chung YS, Son JK, Choi B, Park JB, Chang J, Kim SJ. Transplantation of human spleen into immunodeficient NOD/SCID 
IL2R $\gamma$ (null) mice generates humanized mice that improve functional B cell development. Clin Immunol. 2015;161(2):308-315. 18. Steiniger BS. Human spleen microanatomy: why mice do not suffice. Immunology. 2015;145(3):334-346.

19. Pearse G. Normal structure, function and histology of the thymus. Toxicol Pathol. 2006;34(5):504-514.

20. Steiniger B, Barth P, Hellinger A. The perifollicular and marginal zones of the human splenic white pulp : do fibroblasts guide lymphocyte immigration? Am J Pathol. 2001;159(2):501-512.

21. Greenblatt MB, et al. Graft versus host disease in the bone marrow, liver and thymus humanized mouse model. PLoS One. 2012;7(9):e44664

22. Itoi M, Tsukamoto N, Yoshida H, Amagai T. Mesenchymal cells are required for functional development of thymic epithelial cells. Int Immunol. 2007;19(8):953-964.

23. Nitta T, Murata S, Ueno T, Tanaka K, Takahama Y. Thymic microenvironments for T-cell repertoire formation. Adv Immunol. 2008;99:59-94.

24. Kim BM, Miletich I, Mao J, McMahon AP, Sharpe PA, Shivdasani RA. Independent functions and mechanisms for homeobox gene Barx1 in patterning mouse stomach and spleen. Development. 2007;134(20):3603-3613.

25. Hale LP. Histologic and molecular assessment of human thymus. Ann Diagn Pathol. 2004;8(1):50-60.

26. Hua S, et al. Potential role for HIV-specific CD38-/HLA-DR+ CD8+ T cells in viral suppression and cytotoxicity in HIV controllers. PLoS ONE. 2014;9(7):e101920.

27. Willard-Mack CL. Normal structure, function, and histology of lymph nodes. Toxicol Pathol. 2006;34(5):409-424.

28. Velásquez-Lopera MM, Correa LA, García LF. Human spleen contains different subsets of dendritic cells and regulatory T lymphocytes. Clin Exp Immunol. 2008;154(1):107-114.

29. Melkus MW, et al. Humanized mice mount specific adaptive and innate immune responses to EBV and TSST-1. Nat Med. 2006;12(11):1316-1322.

30. Nicholas KJ, et al. Multiparameter analysis of stimulated human peripheral blood mononuclear cells: A comparison of mass and fluorescence cytometry. Cytometry A. 2016;89(3):271-280.

31. Kaminski DA, Wei C, Qian Y, Rosenberg AF, Sanz I. Advances in human B cell phenotypic profiling. Front Immunol. 2012;3:302.

32. Autissier P, Soulas C, Burdo TH, Williams KC. Evaluation of a 12-color flow cytometry panel to study lymphocyte, monocyte, and dendritic cell subsets in humans. Cytometry A. 2010;77(5):410-419.

33. Shultz LD, Ishikawa F, Greiner DL. Humanized mice in translational biomedical research. Nat Rev Immunol. 2007;7(2):118-130.

34. McMichael AJ, Borrow P, Tomaras GD, Goonetilleke N, Haynes BF. The immune response during acute HIV-1 infection: clues for vaccine development. Nat Rev Immunol. 2010;10(1):11-23.

35. Denton PW, et al. Systemic administration of antiretrovirals prior to exposure prevents rectal and intravenous HIV-1 transmission in humanized BLT mice. PLoS One. 2010;5(1):e8829.

36. Denton PW, et al. Generation of HIV latency in humanized BLT mice. J Virol. 2012;86(1):630-634

37. Swenson LC, et al. Comparative performances of HIV-1 RNA load assays at low viral load levels: results of an international collaboration. J Clin Microbiol. 2014;52(2):517-523.

38. International AIDS Society Scientific Working Group on HIV Cure, et al. Towards an HIV cure: a global scientific strategy. Nat Rev Immunol. 2012;12(8):607-614.

39. Li JZ, et al. The size of the expressed HIV reservoir predicts timing of viral rebound after treatment interruption. AIDS. 2016;30(3):343-353

40. Gupta P, Sanyal A, Mailliard RB. TZA: a novel assay for measuring the latent HIV-1 reservoir. Expert Rev Mol Diagn. 2017;17(12):1033-1035.

41. Sanyal A, et al. Novel assay reveals a large, inducible, replication-competent HIV-1 reservoir in resting CD4+ T cells. Nat Med 2017;23(7):885-889.

42. Nolan DJ, et al. The Spleen Is an HIV-1 Sanctuary During Combined Antiretroviral Therapy. AIDS Res Hum Retroviruses. 2018;34(1):123-125.

43. Sanchez JL, et al. Lymphoid fibrosis occurs in long-term nonprogressors and persists with antiretroviral therapy but may be reversible with curative interventions. J Infect Dis. 2015;211(7):1068-1075.

44. Schacker TW, et al. Lymphatic tissue fibrosis is associated with reduced numbers of naive CD4+ T cells in human immunodeficiency virus type 1 infection. Clin Vaccine Immunol. 2006;13(5):556-560.

45. Zeng M, Haase AT, Schacker TW. Lymphoid tissue structure and HIV-1 infection: life or death for T cells. Trends Immunol. 2012;33(6):306-314.

46. Schacker TW. Defining success with antiretroviral therapy. JAMA Intern Med. 2015;175(1):99-100.

47. Banga R, et al. PD-1(+) and follicular helper T cells are responsible for persistent HIV-1 transcription in treated aviremic individuals. Nat Med. 2016;22(7):754-761.

48. Lavin Y, Mortha A, Rahman A, Merad M. Regulation of macrophage development and function in peripheral tissues. Nat Rev Immunol. 2015;15(12):731-744.

49. Junt T, Scandella E, Ludewig B. Form follows function: lymphoid tissue microarchitecture in antimicrobial immune defence. Nat Rev Immunol. 2008;8(10):764-775.

50. Gieseck RL, Wilson MS, Wynn TA. Type 2 immunity in tissue repair and fibrosis. Nat Rev Immunol. 2018;18(1):62-76.

51. Wang M, et al. Humanized mice in studying efficacy and mechanisms of PD-1-targeted cancer immunotherapy. FASEB J. 2018;32(3):1537-1549.

52. Kozlowska AK, Kaur K, Topchyan P, Jewett A. Novel strategies to target cancer stem cells by NK cells; studies in humanized mice. Front Biosci (Landmark Ed). 2017;22:370-384.

53. Spengler JR, et al. Severity of Disease in Humanized Mice Infected With Ebola Virus or Reston Virus Is Associated With Magnitude of Early Viral Replication in Liver. J Infect Dis. 2017;217(1):58-63.

54. Jaiswal S, et al. Dengue virus infection induces broadly cross-reactive human IgM antibodies that recognize intact virions in humanized BLT-NSG mice. Exp Biol Med (Maywood). 2015;240(1):67-78. 
55. Frias-Staheli N, et al. Utility of humanized BLT mice for analysis of dengue virus infection and antiviral drug testing. $J$ Virol. 2014;88(4):2205-2218.

56. Jaiswal S, et al. Enhanced humoral and HLA-A2-restricted dengue virus-specific T-cell responses in humanized BLT NSG mice. Immunology. 2012;136(3):334-343.

57. Mota J, Rico-Hesse R. Dengue virus tropism in humanized mice recapitulates human dengue fever. PLoS One. 2011;6(6):e20762.

58. Kuruvilla JG, Troyer RM, Devi S, Akkina R. Dengue virus infection and immune response in humanized RAG2(-/-)gamma(c) (-/-) (RAG-hu) mice. Virology. 2007;369(1):143-152.

59. Bente DA, Melkus MW, Garcia JV, Rico-Hesse R. Dengue fever in humanized NOD/SCID mice. J Virol. 2005;79(21):13797-13799.

60. Lee EK, et al. Effects of lymphocyte profile on development of EBV-induced lymphoma subtypes in humanized mice. Proc Natl Acad Sci USA. 2015;112(42):13081-13086.

61. Whitehurst CB, Li G, Montgomery SA, Montgomery ND, Su L, Pagano JS. Knockout of Epstein-Barr virus BPLF1 retards B-cell transformation and lymphoma formation in humanized mice. MBio. 2015;6(5):e01574-e01515.

62. Wang LX, et al. Humanized-BLT mouse model of Kaposi's sarcoma-associated herpesvirus infection. Proc Natl Acad Sci USA 2014;111(8):3146-3151.

63. Prince A, Wang H, Kitur K, Parker D. Humanized Mice Exhibit Increased Susceptibility to Staphylococcus aureus Pneumonia. J Infect Dis. 2017;215(9):1386-1395.

64. Tseng CW, et al. Increased Susceptibility of Humanized NSG Mice to Panton-Valentine Leukocidin and Staphylococcus aureus Skin Infection. PLoS Pathog. 2015;11(11):e1005292.

65. Lynch HE, Goldberg GL, Chidgey A, Van den Brink MR, Boyd R, Sempowski GD. Thymic involution and immune reconstitution. Trends Immunol. 2009;30(7):366-373.

66. Sempowski GD, et al. Leukemia inhibitory factor, oncostatin M, IL-6, and stem cell factor mRNA expression in human thymus increases with age and is associated with thymic atrophy. J Immunol. 2000;164(4):2180-2187.

67. Hale LP. Histologic and molecular assessment of human thymus. Ann Diagn Pathol. 2004;8(1):50-60.

68. Simu G, Bancu VE, Macavei I, Fazekas A, Tohati MT. Microscopic patterns in surgically removed spleens. Rom J Morphol Embryol. 1991;37(1-2):81-86.

69. Mariani R, et al. Mouse-human heterokaryons support efficient human immunodeficiency virus type 1 assembly. $J$ Virol. 2001;75(7):3141-3151

70. Cecilia D, et al. Neutralization profiles of primary human immunodeficiency virus type 1 isolates in the context of coreceptor usage. J Virol. 1998;72(9):6988-6996.

71. Biswas N, et al. ADAR1 is a novel multi targeted anti-HIV-1 cellular protein. Virology. 2012;422(2):265-277.

72. Sanyal A, et al. Novel assay reveals a large, inducible, replication-competent HIV-1 reservoir in resting CD4+ T cells. Nat Med. 2017;23(7):885-889. 